\title{
Single Carrier Frequency Domain Equalization with Space-Time Trellis Codes
}

\author{
Ibukunoluwa Adetutu Adebanjo', Yekeen Olajide Olasoji ${ }^{1}$, Michael Olorunfunmi Kolawole $^{2}$ \\ ${ }^{1}$ Department of Electrical and Electronics Engineering, The Federal University of Technology, Akure, Nigeria \\ ${ }^{2}$ Jolade Strategic Environmental and Engineering Consultants, Melbourne, Australia \\ Email: adetutu.adebanjo@gmail.com,yoolasoji@futa.edu.ng,m.kolawole@jolade.com.au
}

How to cite this paper: Adebanjo, I.A., Olasoji, Y.O. and Kolawole, M.O. (2017) Single Carrier Frequency Domain Equalization with Space-Time Trellis Codes. Communications and Network, 9, 164-171. https://doi.org/10.4236/cn.2017.93011

Received: April 4, 2017

Accepted: August 5, 2017

Published: August 9, 2017

Copyright ( 92017 by authors and Scientific Research Publishing Inc. This work is licensed under the Creative Commons Attribution-NonCommercial International License (CC BY-NC 4.0). http://creativecommons.org/licenses/by-nc/4.0/ (c) (i) \&) Open Access

\begin{abstract}
Orthogonal Frequency Division Multiplexing (OFDM) is readily employed in wireless communication to combat the intersymbol interference (ISI) effect with limited success because as the capacity of MIMO systems increases, other destructive effects affect the propagation channels and/or overall system performance. As such, research interest has increased, on how to improve performance in the mediums where fading and ISI permeate, working on several combinatorial techniques to achieving improved effective throughput. In this study, we propose a combined model of the Space-Time Trellis Code (STTC) and Single-Carrier Frequency Domain Equalization (SC-FDE) to mitigate multiple-fading and interference effects. We present analytical performance results for the combined model over spatially correlated Rayleigh fading channels. We also show that it is beneficial to combine coding with equalization at the system's receiving-end ensuring overall performance: a better performance over the traditional space-time trellis codes.
\end{abstract}

\section{Keywords}

Diversity, ISI, MMSE, STTC, Fading, SC-FDE

\section{Introduction}

Wireless communication is constantly expanding in scope, complexity, and high demand of data usage thereby boosting its research profile. As demand rises, there is a prevailing need to address multipath fading and intersymbol interference (ISI) [1]. In mobile outdoors environment, where a characterizing-mark is the absence of the line-of-sight, multipath fading and ISI influence is unavoidable. To mitigate this effect, a multicarrier scheme-OFDM-has constantly been incorporated in mobile designs. OFDM performs Fast Fourier 
Transform (FFT) operation on transmitted and received signals over parallel subcarrier thereby reducing or eliminating interference [2] [3]. Although OFDM has proven limited success in combating ISI and multipath fading, it has inherent drawback because as the capacity of MIMO systems increases, other destructive effects affect the propagation channels and/or overall system performance [4]. Equalization techniques (time and frequency domain), as well as space-diversity techniques, are being applied to mitigate these effects [5] [6] [7] [8]. As such, research interest has increased, on how to improve performance in the mediums where slow and fast fading and ISI permeate, working on several combinatorial techniques to achieving improved effective throughput. In this study, space-time coding technique (STTC) was combined with SC-FDE using the rank-determinant criteria. SC-FDE has a similar performance as OFDM [9] [10], however SC-FDE has a lower average peak-to-power ratio than OFDM, making its dynamic range of power amplification lesser than OFDM. The background information on STTC and SC-FDE was examined, as well as the effect on the overall performance when combining coding with equalization at the receiver.

\section{Background Space-Time Codes Theory}

Tarokh et al. [8] pioneered the concept of space-time coding. Data sequence is propagated in space over $n_{t}$ number of transmit antennas, and in time, over $t$ symbol periods yielding $\left(n_{t} \times t\right)$ codeword and coding rate that is a fraction of the number of sequence over the symbol period.

\section{Performance Analysis of Space-Time Codes}

The performance of any wireless communication system is a measure of the percentage number of bits in error. The performance index of space-time-code is determined by the BER performance: a measure of the distance attributes of the code [11]. The BER performance analysis of space-time code can be examined by considering the Pairwise Error Probability (PEP). Generally, in the design of space-time codes, quasi-static fading model of MIMO is usually adopted [12].

Considering a space-time coding system with $n_{t}$ transmit antenna and $n_{R}$ receive antenna over spatially correlated Rayleigh fading channel. The received sequence is denoted by

$$
R=H X+N
$$

where $X, H$, and $N$ denote the transmitted signal, channel matrix, and additive white noise, respectively, and corresponding dimensions $n_{t} \times T$ where $T$ is the symbol durations through $n_{t}$ transmit antennas, $n_{t} \times n_{t}$, and $n_{r} \times 1$. Suppose the channel is known to the receiver, and a codeword $c=c_{1}^{1} c_{1}^{2} \cdots c_{1}^{n} c_{2}^{1} c_{2}^{2} \cdots c_{2}^{n} \cdots c_{l}^{1} c_{l}^{2} \cdots c_{l}^{n}$ was transmitted and the receiver decides erroneously in favor of signal $e=e_{1}^{1} e_{1}^{2} \cdots e_{1}^{n} e_{2}^{1} e_{2}^{2} \cdots e_{2}^{n} \cdots e_{l}^{1} e_{l}^{2} \cdots e_{l}^{n}$, then a difference matrix can be obtained as [8] [13]: 


$$
B(c, e)=\left[\begin{array}{cccc}
e_{1}^{1}-c_{1}^{1} & e_{2}^{1}-c_{2}^{1} & \cdots & e_{1}^{1}-c_{l}^{1} \\
e_{1}^{2}-c_{1}^{2} & e_{2}^{2}-c_{2}^{2} & \cdots & e_{1}^{2}-c_{l}^{2} \\
\vdots & \vdots & \ddots & \vdots \\
e_{1}^{n}-c_{1}^{2} & e_{2}^{n}-c_{2}^{n} & \cdots & e_{l}^{n}-c_{l}^{n}
\end{array}\right]
$$

In the Rayleigh fading channel, the Ricean factor equals to zero. Therefore, the average pairwise error probability (PEP, or simply $P(C \rightarrow E)$ ) between two arbitrary codewords $C$ and $E$ over independent and identically distributed Rayleigh fading channels is written as [7]

$$
\begin{aligned}
P(C & \rightarrow E)=\frac{1}{\pi} \int_{0}^{\pi / 2}\left(\operatorname{det}\left(I_{T n_{r} n_{t}}+\eta \vartheta \Delta\right)\right)^{-1} \mathrm{~d} \beta \\
& =\frac{1}{\pi} \int_{0}^{\pi / 2} \prod_{i=1}^{r(\vartheta \Delta)}\left(1+\eta \lambda_{i}(\vartheta \Delta)\right)^{-1} \mathrm{~d} \beta
\end{aligned}
$$

where

$\vartheta$ is the spatio-temporal correlation matrix;

$r(\vartheta \Delta)$ is the rank of $\vartheta \Delta$;

$\eta$ is the effective signal-to-noise-ratio, SNR;

$\beta$ is intergrated over the maximum at $\beta=\frac{\pi}{2}$. Equation (4) is obtained from the Gaussian Q-function.

Ensuring that the code is not rank-deficient, suppose the Rayleigh fading channel is stable per frame, Equation (4) can thus be reduced to [14]

$$
P(C \rightarrow E)=\frac{1}{\pi} \int_{0}^{\frac{\pi}{2}} \prod_{i=1}^{r\left(C_{R}\right)}\left(1+\eta \lambda_{i}\left(C_{R}\right)\right)^{-1} \mathrm{~d} \beta
$$

when $R=R_{r} \otimes R_{t} . R_{r}$ and $R_{t}$ are the receive and transmit correlation matrices, respectively. At high SNR, Equation (5) results in

$$
P(C \rightarrow E) \cong \frac{1}{\pi} \int_{0}^{\frac{\pi}{2}} \eta^{-r\left(C_{R}\right)} \prod_{i=1}^{r\left(C_{R}\right)} \lambda_{i}^{-1}\left(C_{R}\right)^{-1} \mathrm{~d} \beta
$$

In space-time coding, for slow fades, the rank-determinant criterion is usually used [9] [15]. So,

$$
P(C \rightarrow E) \cong \prod_{i=1}^{r\left(C_{R}\right)}\left(1+\frac{p}{4} \lambda_{i}\left(C_{R}\right)\right)^{-n_{r}}
$$

However, for high SNR using the rank-determinant criterion, Equation (7) leads to

$$
P(C \rightarrow E) \leq\left(\frac{p}{4}\right)^{-n_{r} r\left(C_{R}\right)} \prod_{i=1}^{r\left(C_{R}\right)} \lambda_{i}^{-n_{r}}\left(C_{R}\right)
$$

where $\lambda_{i}^{-n_{r}}\left(C_{R}\right)$ is the $n_{r}$ th power of $\lambda_{i}\left(C_{R}\right)$.

Maximization of the rank of the error matrix $C_{R}$ gives the diversity gain. Coding gain is obtained by maximizing the $\prod_{i=1}^{r\left(C_{R}\right)} \lambda_{i}^{-n_{r}}\left(C_{R}\right)$ quantity.

\section{System Model of STTC and SC-FDE}

Consider a single carrier block transmission after serial binary bits being mapped 
into parallel bits, and the cyclic prefix (CP) inserted into the blocks of bits, as shown in Figure 1. The prefixed data stream is grouped (L-blocked) into serial combination and sent through the channel. This explains the simplicity of the single-carrier block transmission at the transmit side [10] [16]. In combining with STTC, the output streams from the encoder are appended with the cyclic prefix, which must be longer than the channel delay spread. These $N+L$ streams of data are sent through the wireless channel after parallel conversion.

Assuming $s(t)$ was encoded into $n_{t}$ streams of data $x(t)$ expressed as

$$
X(t)=\left[X_{0} X_{1} \cdots X_{t} \cdots\right]=\left[\begin{array}{ccccc}
x_{0}^{1} & x_{1}^{1} & \cdots & x_{t}^{1} & \cdots \\
x_{0}^{2} & x_{1}^{2} & \cdots & x_{t}^{2} & \cdots \\
\vdots & \vdots & \ddots & \vdots & \cdots \\
x_{0}^{N_{T}} & x_{1}^{N_{T}} & \cdots & x_{t}^{N_{T}} & \cdots
\end{array}\right]
$$

and when appended with $\mathrm{CP}$ of length $L$ becomes

$$
\left[\begin{array}{ccccc}
x_{t-1}^{1} & x_{t}^{1} & x_{0}^{1} & x_{1}^{1} & \ldots \\
x_{t-1}^{2} & x_{t}^{2} & x_{0}^{2} & x_{1}^{2} & \ldots
\end{array}\right]
$$

At the receiver, the received signal is given by

$$
r_{t}^{j}=\sum_{i=1}^{n} h_{i j}(t) x_{t}^{i}(t)+\eta_{t}^{j}
$$

where $j(=1,2, \cdots)$ is the number of receive antennas, $\eta_{t}^{j}$ is the effective SNR additive white Gaussian noise at time $t$ of antenna $j ; x_{t}^{i}(t)$ is the transmitted signal from $i$ number of transmit antenna; $h_{i j}(t)$ is the complex channel coefficient. By considering the channel to be slowly fading, then Equation (11) can be written as

$$
R=H_{C P}\left[X_{i}\right]+\eta
$$

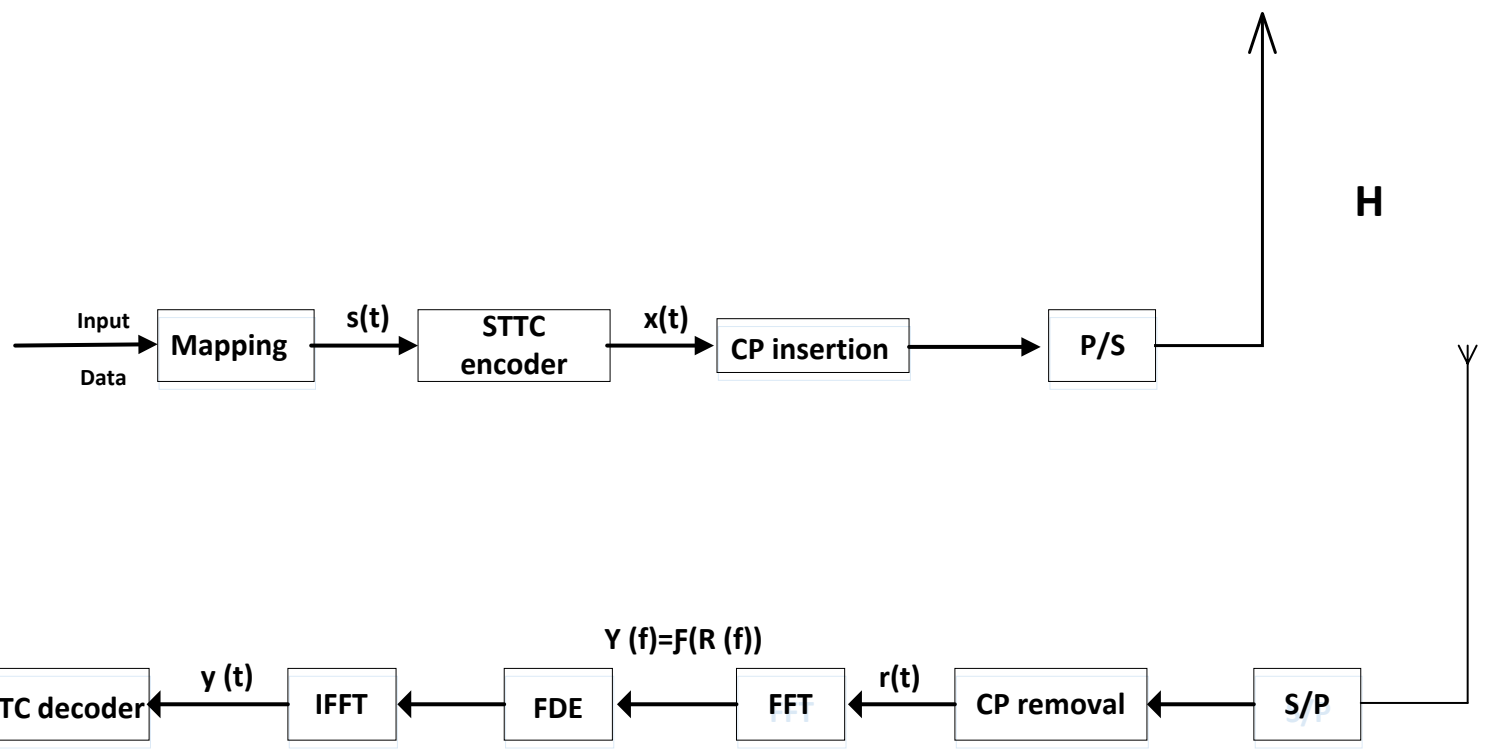

Figure 1. A single-carrier Space Time Trellis Code (STTC) model. 
where $H_{C P}$ is a block-wise circulant square matrix of size $T \times T$ in the form:

$$
H_{C P}=\left[\begin{array}{ccccc}
H(0) & H(L-1) & H(L-2) & \cdots & H(1) \\
H(1) & H(0) & H(L-1) & \cdots & H(2) \\
\vdots & \vdots & \vdots & \ddots & \vdots \\
H(L-1) & H(L-2) & \cdots & \ldots & H(0)
\end{array}\right]
$$

Obtaining the singular value decomposition, $H_{C P}$ becomes $\mathcal{F}^{H} \Lambda_{C P} \mathcal{F}$, where $\Lambda_{C P}$ is a diagonal matrix whose elements are obtained by a block-wise FFT of $[H(0) H(1) \cdots H(L-1)], \mathcal{F}$ is the Discrete Fourier Transform (DFT) matrix.

$$
\Lambda_{C P}=\sum_{l=0}^{L-1} H(l) \mathrm{e}^{-j \frac{2 \pi}{T} k l}
$$

The eigenvectors of $H_{C P}$ are independent of channel matrices $H(I)$ [7], $\Lambda_{C P}$ bears the full information of the channel which the FFT operation requires. Applying FFT operation on the received vector, we have

$$
Y(f)=\mathcal{F}[R(f)]=\Lambda_{C P}[X(f)+[\eta(f)]
$$

Performing equalization using the minimum mean square, which minimizes the mean square error between the estimated and received symbols, and assuming an MMSE equalizer coefficient, we write Equation (15) as

$$
y=\sqrt{\rho} W H_{C P} X+\eta_{w}
$$

where $\rho$ is the signal-to-noise ratio, $W$ is the equalizer coefficient, and $\eta_{w}$, noise as a result of equalization. Performing inverse FFT on Equation (16) results

$$
F^{-1}(y)=F^{-1} \sqrt{\rho} W H_{C P} F^{-1} x+F^{-1} \eta_{w}
$$

which is consistent with [17].

\section{Simulation Results}

The Bit Error Rate (BER) evaluation of the combined model over spatially correlated Rayleigh fading channels was done. Table 1 shows the parameters used for simulation.

Table 1. Major simulation parameters.

\begin{tabular}{cc} 
MIMO channel type & 3GPP ITU Pedestrian A \\
Fading distribution & Rayleigh \\
FFT size & 512 \\
Channel bandwidth & $5 \mathrm{MHz}$ \\
Cyclic prefix length & 40 \\
Modulation scheme & QPSK \\
Antenna configuration & $2 \times 2$ \\
Channel coding & None \\
Channel estimation and equalization & Minimum Mean Square Error (MMSE) \\
\hline
\end{tabular}


It was observed from the results shown in Figure 2 that for the same conditions and low SNR; that is, SNR $\leq 15 \mathrm{~dB}$, the BER performances of basic STTC and STTC-FDE using MMSE systems were virtually the same. However, BER performance improves marginally as the channel becomes noisy for STTC-FDE as compared to the basic STTC. This result demonstrates that although it is possible to achieve higher level of coding gain with trellis coding alone, but in wireless communication systems targeting at broadband and mobile transmissions commonly face the challenge of fading channels that are both time and frequency selective, the use of space-time coding with equalization in the frequency domain improves the performance of the systems.

Figure 3 shows the average pairwise error probability (PEP) of basic STTC and STTC-FDE using MMSE. Minimum PEP results in space-time code when the Euclidean distance is maximized. It was observed that at low SNR, the PEP was high in both systems. At high SNR (i.e. SNR $\geq 12 \mathrm{~dB}$ ), the PEP was reduced for both STTC-MMSE. The diversity order played a key role in obtaining the PEP. It was observed that the plot of STTC-MMSE gradually slopes down from a low SNR to high SNR. For the STTC, PEP gap was maintained evenly. At BER = $10^{-4}$, the PEP obtained for STTC-MMSE had a gain of $6 \mathrm{~dB}$ compared to the STTC.

The operation of having to decode the transmitted symbol in the time domain could explain the gain. The probability that the decoder would select an erroneous signal was low due to the fact that equalization and the FFT/IFFT operations were carried out before the STTC decoding. More studies are continuing on large antenna configuration.

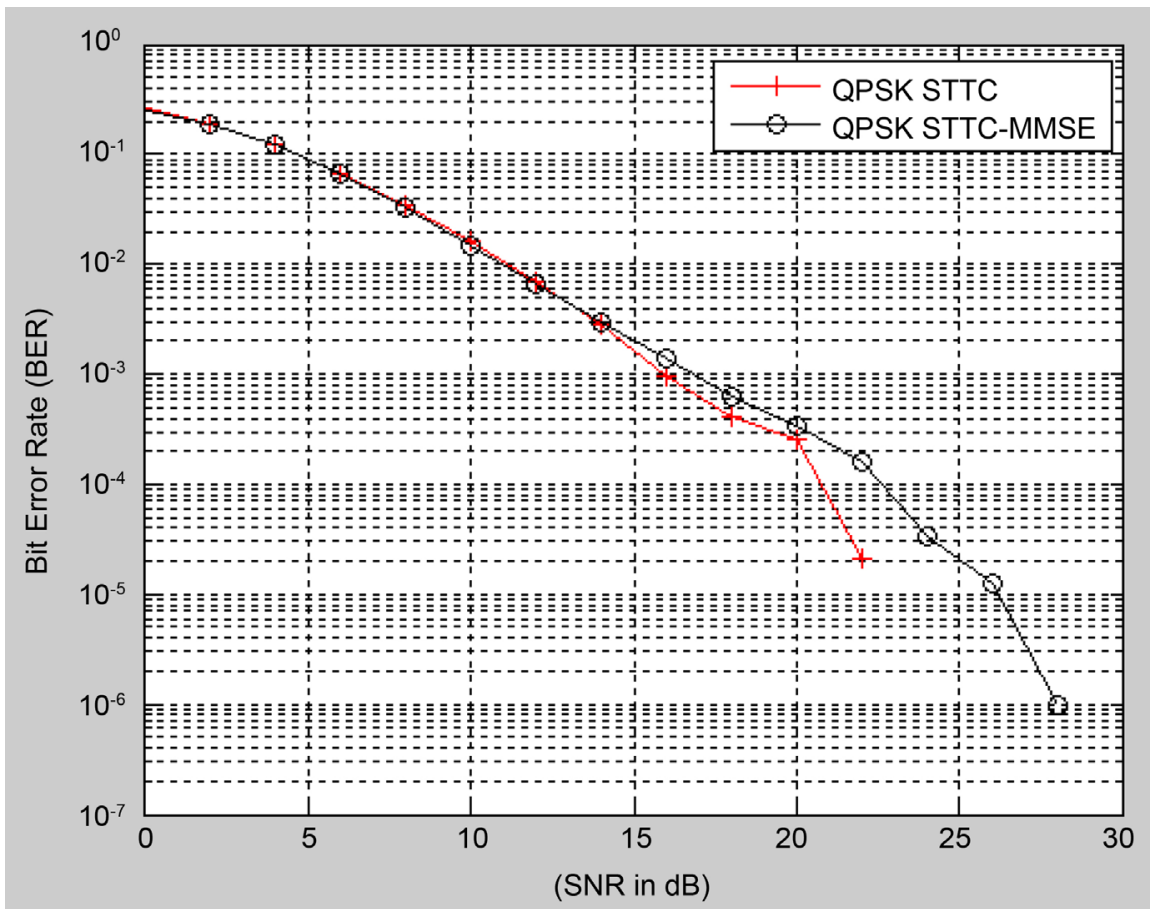

Figure 2. Average BER-SNR performance of STTC and STTC-MMSE. 


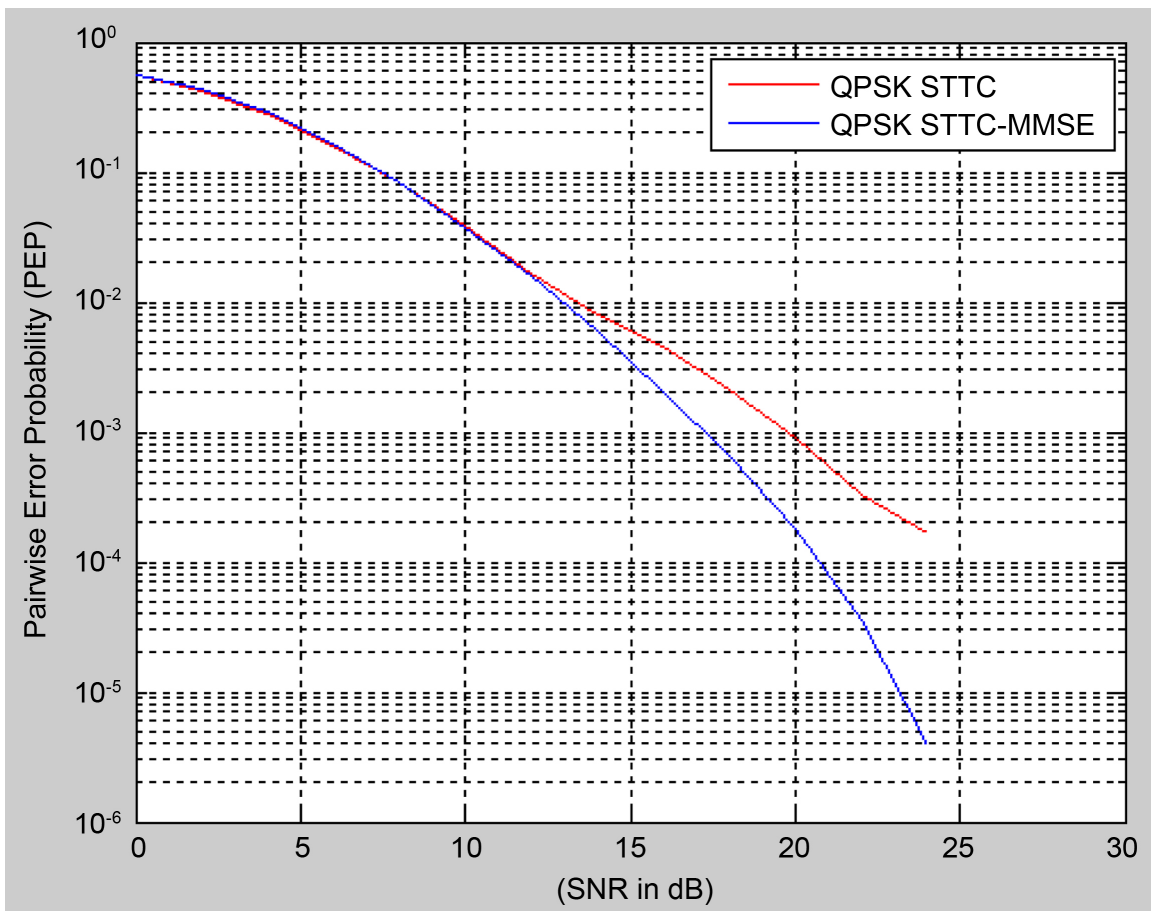

Figure 3. Average PEP-SNR performance plot of STTC and STTC-MMSE.

\section{Conclusion}

This paper has examined the performance of wireless communication when space-time trellis code is combined with Equalization in single-carrier transmission. In combining the two techniques, it was observed that BER increases marginally for STTC-MMSE compared to the basic STTC. Pairwise Error Probability shows an improved performance of STTC-MMSE over the traditional STTC. The error analysis obtained shows the viability of implementing a combination of diversity with frequency equalization in wireless communication.

\section{References}

[1] Coon, J. and Beach, M. (2002) An Investigation of MIMO Single-Carrier Frequency-Domain MMSE Equalization. Centre for Communications Research, University of Bristol.

[2] Falconer, D.D. and Ariyavisitakul, S.L. (2002) Broadband Wireless using Single Carrier and Frequency Domain Equalization. International Conference on Wireless Personal Multimedia Communications, 27-36. https://doi.org/10.1109/WPMC.2002.1088127

[3] Gmini, L.J. (1985) Analysis and Simulation of a Digital Mobile Channel using Orthogonal Frequency Division Multiplexing. IEEE Transaction on Communication, 33, 665-675. https://doi.org/10.1109/TCOM.1985.1096357

[4] Kolawole, M.O. (2013) Satellite Communication Engineering. CRC Press, New York.

[5] Sugiura, S., Chen, S. and Hanzo, L. (2012) A Universal Space-Time Architecture for Multiple-Antenna Aided Systems. IEEE Communications Surveys and Tutorials, 14, 401-420. https://doi.org/10.1109/SURV.2011.041911.00105 
[6] Foschini, G.J. and Gans, M.J. (1998) On Limits of Wireless Communications in a Fading Environment When using Multiple Antennas. Wireless Personal Communication, 6, 311-335. https://doi.org/10.1023/A:1008889222784

[7] Clerckx, B. and Oestges, C. (2007) MIMO Wireless Communication: From RealWorld Propagation to Space-Time Code Design. Academic Press, Oxford.

[8] Tarokh, V., Seshadri, N. and Calderbank, A.R. (1998) Space-Time Codes for High Data Rate Wireless Communication: Performance Criterion and Code Construction. IEEE Transactions on Information Theory, 44, 744-765. https://doi.org/10.1109/18.661517

[9] Pancaldi, F., Vitetta, G.M., Kalbasi, R., Al-Dhahir, N., Uysal, M. and Mheidat, H. (2008) Single-Carrier Frequency Domain Equalization-A Focus on Wireless Applications. IEEE Signal Processing Magazine, 25, 37-56.

https://doi.org/10.1109/MSP.2008.926657

[10] Yune, T.N., Seol, D.Y., Kim, D. and Im, G.H. (2010) Single-Carrier Frequency Domain Equalization for Broadband Cooperative Communications. Cooperative Communications for Improved Wireless Network Transmission: Framework for Virtual Antenna Array Applications, 399.

[11] Sibille, A., Oestges, C. and Zanella, A. (2011) MIMO: From Theory to Implementation. Academic Press, Oxford.

[12] Chockalingham, A. and Rajan, B.S. (2014) Large MIMO Systems. Cambridge University Press, New York.

[13] Rassool, B.A., Heliot, F., Revelly, L., Dohler, M., Nakhai, R. and Aghvami, H. (2003) Fast Search Techniques for Obtaining Space-Time Trellis Codes for Rayleigh Fading Channels and Its Performance in CDMA Systems. Proceedings of IEEE Vehicular Technology Conference, 1, 66-69. https://doi.org/10.1109/VETECS.2003.1207503

[14] Hong, Y. and Fàbregas, A.G. (2006) New Space-Time Trellis Codes for Slow Fading Channels. Proceedings of IEEE Vehicular Technology Conference, 3, 1492-1496. https://doi.org/10.1109/VETECS.2006.1683084

[15] Jafarkhani, H. (2005) Space-Time Coding, Theory and Practice. Cambridge University Press, New York. https://doi.org/10.1017/CBO9780511536779

[16] Falconer, D., Ajra, H., Hasan, Z., Ariyavisitakul, S.L., Benyamin-Seeyar, A. and Eidson, B. (2002) Frequency Domain Equalization for Single-Carrier Wireless Systems. IEEE Communications Magazine, 40, 58-66. https://doi.org/10.1109/35.995852

[17] Islam, S. (2014) BER Analysis of Various Channel Equalization Schemes of a QOSTBC Encoded OFDM. International Journal of Communication Networks and Information Security, 3, 30-36. https://doi.org/10.1504/IJSN.2014.059325 
Submit or recommend next manuscript to SCIRP and we will provide best service for you:

Accepting pre-submission inquiries through Email, Facebook, LinkedIn, Twitter, etc. A wide selection of journals (inclusive of 9 subjects, more than 200 journals)

Providing 24-hour high-quality service

User-friendly online submission system

Fair and swift peer-review system

Efficient typesetting and proofreading procedure

Display of the result of downloads and visits, as well as the number of cited articles Maximum dissemination of your research work

Submit your manuscript at: http://papersubmission.scirp.org/

Or contact cn@scirp.org 